Musées, Patrimoine et Culture scientifiques et techniques

\title{
L'évolution du rôle des réserves muséales : les réserves délocalisées du musée de l'Armée
}

The evolution of the role of museum storerooms: the Army's relocated museum storerooms

Sandrine Beaujard-Vallet

\section{(2) OpenEdition}

Journals

Édition électronique

URL : http://journals.openedition.org/ocim/975

DOI : 10.4000/ocim.975

ISSN : 2108-646X

Éditeur

OCIM

Édition imprimée

Date de publication : 1 novembre 2011

Pagination : 11-15

ISSN : 0994-1908

Référence électronique

Sandrine Beaujard-Vallet, «L'évolution du rôle des réserves muséales : les réserves délocalisées du musée de l'Armée », La Lettre de l'OCIM [En ligne], 138 | 2011, mis en ligne le 01 novembre 2013, consulté le 01 mai 2019. URL : http://journals.openedition.org/ocim/975; DOI : 10.4000/ocim.975 
du patrimoine. Cette évolution est telle que sur la question du rôle des réserves, il convient de parler d'une véritable rupture avec le passé. La réserve est devenue un outil au service des objets, de leur conservation et de leur diffusion. La question de la création ou de la rénovation des réserves reste un sujet délicat à plaider auprès des administrations de tutelle. Le sujet n'étant pas visible par le public dans la plupart des cas (à l'exception des réserves visibles ou visitables comme celles du musée des Arts et Métiers. ou encore la réserve des armes et armures anciennes au musée de l'Armée), il est donc peu médiatique pour les politiques. Heureusement les mentalités évoluent et les responsables scientifiques des collections possèdent les arguments permettant de convaincre. Nous professionnels avons l'obligation de transmettre, d'enrichir, de documenter les objets des collections patrimoniales pour les générations futures. Transmettre, enrichir et conserver efficacement les collections nécessitent d'intégrer de nouvelles fonctions à la réserve et de supprimer définitivement l'image du grenier domestique encore présente dans la conscience collective. Les réserves sont donc toujours un lieu où l'on conserve les objets qui ne sont pas présentés au public, mais elles sont aussi un lieu d'étude pour les chercheurs, historiens, historiens de l'art. C'est un endroit où l'on sélectionne les objets pour les expositions, les prêts, les dépôts. Les objets y sont préparés, conservés et parfois même restaurés. Les réserves sont ainsi devenues le fondement du musée.

\section{Le programme de rénovation du musée de l'Armée}

Le musée de l'Armée est né de la fusion des collections du musée de l'Artillerie et du musée historique de l'Armée. Un décret fixe les conditions de cette fusion, celui du 26 juillet 1905. Fondé sous la Révolution française, le musée de l'Artillerie regroupait les collections du Garde meubles de la Couronne et celles des Princes de Condé. En 1872, le musée de l'Artillerie est installé aux Invalides. Le musée historique de l'Armée a été fondé en 1896 par la Sabretache et son président Édouard Detaille. À cela s'ajoute la nécropole militaire où se trouvent notamment les sépultures de Turenne, Foch, Vauban, Lyautey, Bonaparte.

Le musée vit, depuis la fin des années 1990, une mutation profonde. En 1997-1998, débute un programme de modernisation globale, dénommé ATHENA (Armes, Techniques, Histoire, Emblématiques, Nation, Armée). L'objectif de ce programme de rénovation était de transformer un musée d'objets en musée d'Histoire de l'Armée de Terre depuis le Moyen Âge jusqu'à nos jours.

Le nouveau parcours s'appuie sur trois figures emblématiques des grandes périodes de l'Histoire de France, l'Armée Royale avec Louis XIV ; l'Armée Impériale avec

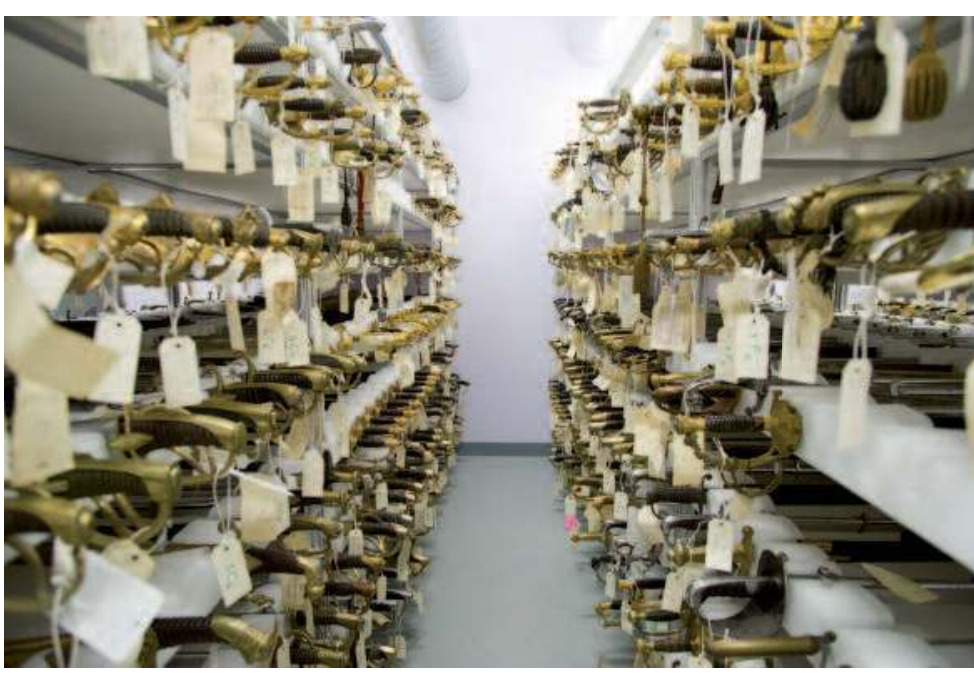

Les réserves : les rateliers pour les armes blanches ๑) Musée de l'Armée/Fanny Reynaud

Napoléon Ier ; l'Armée contemporaine de la République avec Charles de Gaulle. Le rez-de-chaussée de l'aile occidentale de l'Hôtel national des Invalides est consacré depuis décembre 2005 aux armes et armures anciennes, couvrant ainsi, une surface d'exposition de plus de $2500 \mathrm{~m}^{2}$. Depuis le mois de juin 2006, les étages de cette aile sont des espaces dédiés à la troisième République, et aux deux guerres mondiales, sur près de $3500 \mathrm{~m}^{2}$. L'ancienne muséographie présentait les deux conflits mondiaux, mais aussi les petits modèles d'artillerie (salle Gribeauval) et les armées étrangères.

Le programme ATHENA a permis un réaménagement de ces espaces en incluant des salles consacrées à la IIIe République. Durant la même période, les réserves du musée situées dans des caves de l'Hôtel national des Invalides, ont été déménagées et délocalisées en banlieue parisienne, sur un terrain militaire, près de Versailles. Les bâtiments de réserves sont aujourd'hui au nombre de trois et se déploient sur plus de $3500 \mathrm{~m}^{2}$. Enfin en 2009 et 2010, les espaces d'exposition consacrés aux collections couvrant la période historique allant de Louis XIV à Napoléon III, ont été ouverts aux visiteurs après trois années de travaux et de chantier des collections. Une salle de synthèse sur les traditions militaires à partir d'instruments de musique, de figurines historiques et de petits modèles d'artillerie, ainsi que des espaces d'exposition temporaire sur plus de $800 \mathrm{~m}^{2}$ sont actuellement en cours de création. D'autres travaux débutent et concernent l'élaboration d'un centre documentaire, d'ateliers pédagogiques et d'une bibliothèque. Les trois ateliers de restauration du musée sont d'ores et déjà rénovés. 


\section{Du simple lieu de stockage des collections au pôle de conservation du patrimoine}

La place des réserves a été définie par le Conseil International des Musées (ICOM), en 2002, dans son Code de Déontologie.

"L'autorité de tutelle d'un musée a le devoir éthique de maintenir et de développer tous les aspects d'un musée, ses collections et ses services. Surtout, elle a la responsabilité de veiller à ce que toutes les collections qui lui sont confiées soient abritées, conservées et documentées de façon appropriée ".

"L'une des obligations déontologiques essentielles de chaque professionnel de musée est d'assurer une protection et une conservation satisfaisantes des collections et des objets individuels dont l'institution employeuse est responsable. Le but doit être d'assurer, dans la mesure du possible, la transmission des collections aux générations futures en aussi bon état de conservation que possible en égard aux conditions actuelles des connaissances et des ressources. (...) Tous les professionnels de musée qui ont la charge d'objets et de spécimens se doivent de créer et d'entretenir un environnement protecteur pour les collections, qu'elles soient en réserve, en exposition ou en cours de transport. Cette conservation préventive constitue un élément important dans la gestion des risques d'un musée ».

\section{L'aménagement des réserves délocalisées de Versailles-Satory}

La délocalisation des réserves est un choix raisonné survenu en 2002. Plusieurs motifs en sont à l'origine. Ce choix résulte en partie de la nécessité absolue d'évacuer des réserves situées dans les anciennes caves de l'Hôtel national des Invalides et inondables par remontée des nappes phréatiques en cas de crue centennale de la Seine. Dans le contexte général d'amélioration des conditions de conservation des objets de la collection, il est apparu évident d'optimiser les conditions de stockage et de conservation des objets en réserve. Le transfert devait également avoir lieu en raison du redéploiement des locaux techniques des salles d'exposition et de la création de l'Historial Charles de Gaulle, véritable pôle multimédia déployé sur $800 \mathrm{~m}^{2}$ et situé sous la cour de la Valeur, en lieu et place des anciennes réserves.

Dans les contraintes à prendre en compte, l'accès au lieu choisi devait être facilité pour le personnel de la conservation. Une navette a été mise en place pour rejoindre les réserves depuis le site des Invalides.

\section{Un travail d'équipe}

Le ministère de la Défense a d'abord attribué au musée un bâtiment de troupes de $2000 \mathrm{~m}^{2}$. Celui-ci a été entièrement réhabilité pour créer une réserve fonctionnelle. Depuis, deux autres bâtiments de réserves ont été attribués au musée et aménagés. L'un est consacré au stockage de l'armement

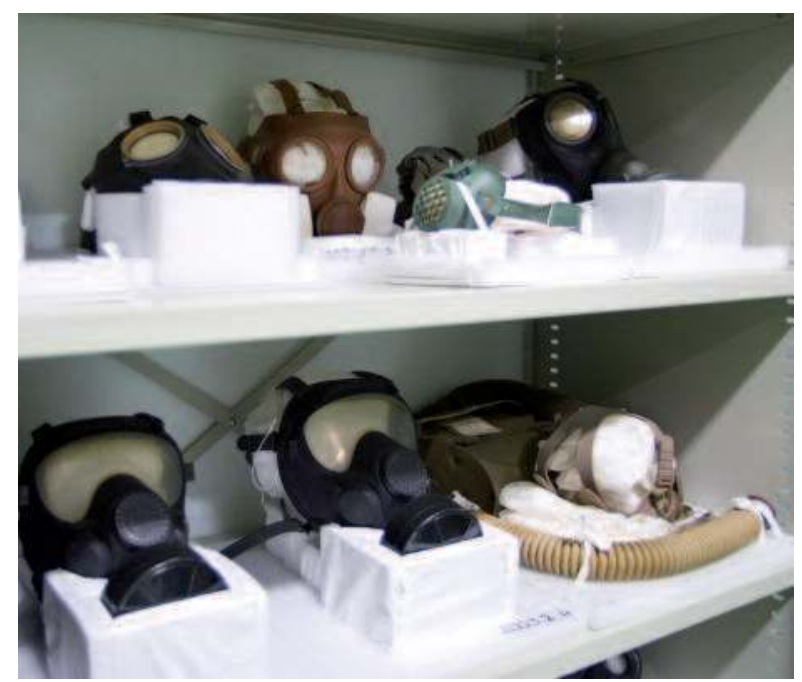

Les réserves : protections individuelles pour les masques à gaz (c) Musée de l'Armée/Fanny Reynaud

portatif, l'autre à celui de l'artillerie lourde. Ils se déploient respectivement sur 400 et $1000 \mathrm{~m}^{2}$.

L'adaptation des bâtiments est un travail qui a rassemblé de nombreux acteurs faisant intervenir plusieurs services du musée. Le travail avec les architectes chargés de la maîtrise d'ouvrage s'est fait en collaboration avec les conservateurs responsables des départements de la conservation et les responsables des services infrastructure et sécurité du musée bien sûr mais, fait suffisamment exceptionnel pour être souligné, avec le régisseur des collections et tout le personnel de la régie, utilisateurs privilégiés de la réserve. L'adéquation entre les matériaux utilisés et les objets stockés ainsi que les choix concernant l'environnement des objets résultent de ces échanges entre l'architecte et le régisseur. Le confort d'utilisation des installations, l'accessibilité aux salles de réserves et la fonctionnalité des espaces de travail sont le fruit de l'association de certains membres du personnel souvent « oubliés » des projets. Il est apparu primordial de demander leur point de vue aux assistants de la régie des collections, aux magasiniers, aux agents chargés de la sécurité et de la sûreté.

\section{L'adaptation et la rénovation} des bâtiments existants

Les bâtiments mis à disposition par le ministère de la Défense sont de natures diverses. Le premier est un ancien bâtiment de troupes, très cloisonné. Le second est une ancienne menuiserie. Le troisième est une ancienne écurie. Les travaux de gros œuvre ont concerné le nouvel agencement, l'isolation par un doublage performant et adapté, la réfection des sols et la pose d'un sol en résine. Concernant le traitement climatique, différentes options ont été choisies en fonction de la destination des bâtiments 
et des matériaux constitutifs des objets à stocker. Après étude des relevés climatiques, il a été décidé par la maitrise d'œuvre et par la maitrise d'ouvrage d'installer une centrale de renouvellement d'air avec filtration sur charbon actif pour l'un des bâtiments disposant d'un vide sanitaire et d'un chauffage par pompe à chaleur. Un autre bâtiment possède une centrale de traitement d'air, ce qui résulte d'une longue période de réflexion et d'hésitation quant à la maintenance difficile et bien connue de ce genre d'installation. L'absence de vide sanitaire, la nécessité de conserver des objets principalement constitués de fer et la présence d'une aire de quarantaine destinée aux traitements par anoxie des collections, a orienté la réflexion vers ce choix. Enfin, le troisième bâtiment est isolé hors-gel pour le stockage des matériels d'artillerie lourde.

\section{Un stockage par typologie d'objets}

Le premier édifice dispose d'une surface de $2000 \mathrm{~m}^{2}$ de stockage et se répartit sur deux niveaux. Il comprend quatre salles de réserves au rez-de-chaussée, un atelier de conservation préventive, ainsi qu'une zone d'emballage et de déballage des objets. Il comprend également huit salles de réserves à l'étage. Les objets sont stockés par typologie et par matériaux constitutifs. Le mobilier est soit métallique recouvert d'une peinture époxy cuite au four, soit en aluminium. La charge au sol étant limitée à $500 \mathrm{~kg} / \mathrm{m}^{2}$ au rez-de-chaussée et $400 \mathrm{~kg} / \mathrm{m}^{2}$ à l'étage, le choix d'un matériau léger était important. Le mobilier est souvent standard, mais certaines spécificités liées au stockage d'objets d'histoire militaire a parfois nécessité la création sur prototypes.

Une réserve équipée d'une centrale de traitement $\mathrm{d}^{\prime}$ air, équipement nécessaire à ses fonctions

La réserve consacrée au stockage de l'armement portatif est équipée d'une centrale de traitement d'air afin de
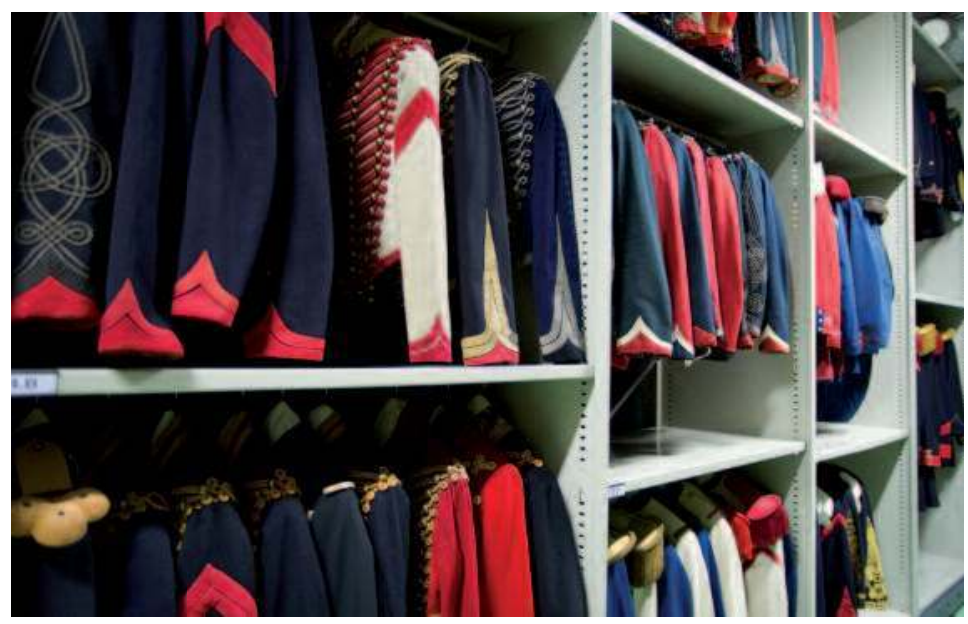

Les réserves : meubles de rangement pour les uniformes (c) Musée de l'Armée/Fanny Reynaud conserver le métal à un taux d'humidité relative bas. Un traitement d'air différencié permet d'obtenir le climat nécessaire à la réussite des traitements par anoxie statique dans l'aire de quarantaine et d'empêcher toute contamination.

\section{Une réserve pour les objets}

\section{lourds et encombrants}

Enfin, la réserve des pièces d'artillerie lourde se déploie sur $800 \mathrm{~m}^{2}$ et garantit la conservation dans un climat hors-gel. Il s'agit d'une ancienne écurie, entièrement rénovée. Les travaux d'isolation ont porté sur le sol, les murs et la toiture. Le sol a également été renforcé pour supporter des charges très lourdes. L'idée était d'ajuster soigneusement les aménagements projetés à la nature des différents objets en évitant de mettre en place des équipements sophistiqués et coûteux qui ne seraient pas nécessaires ainsi que d'exploiter la structure en quatre travées parallèles de l'édifice et en utilisant au mieux les liaisons existantes entre elles.

\section{L'évolution des exigences de la conservation à travers l'expression des besoins}

\section{La fonctionnalité}

Lors de la création des réserves délocalisées, de nombreuses questions visant à élaborer le schéma fonctionnel du lieu ont été posées. La réflexion a porté sur l'opportunité d’y croiser différentes fonctions. Les réserves ont ainsi été équipées d'aires de quarantaine pour les objets risquant d'être infestés ou contaminés, d'un lieu d'étude pour les chercheurs, d'un ou de plusieurs ateliers de conservation préventive, voire de conservation-restauration, d'un laboratoire de prises de vue, d'un local d'emballage et de déballage des objets pour les expositions temporaires, d'un bureau permettant la gestion informatisée des collections (localisations, mouvements).

\section{Une équipe}

Les moyens humains ont aussi été entièrement repensés. Le recrutement de professionnels formés à la conservation préventive notamment, a permis d'apporter de nouvelles compétences au sein de l'établissement et ainsi de faciliter la gestion des réserves. L'équipe est constituée de sept personnels permanents aux tâches spécialisées, comme la surveillance et la sécurité, le magasinage, la gestion des collections, les opérations de conservation préventive... Travail d'équipe et polyvalence sont de mise. L'un des bâtiments est équipé d'un atelier de conservation préventive dans lequel travaille une technicienne de préservation diplômée, sous contrat avec l'établissement.

\section{L'organisation du travail}

Le chantier des collections en réserve a débuté en 2004 et se poursuit. Toutes les opérations d'un chantier de collections 


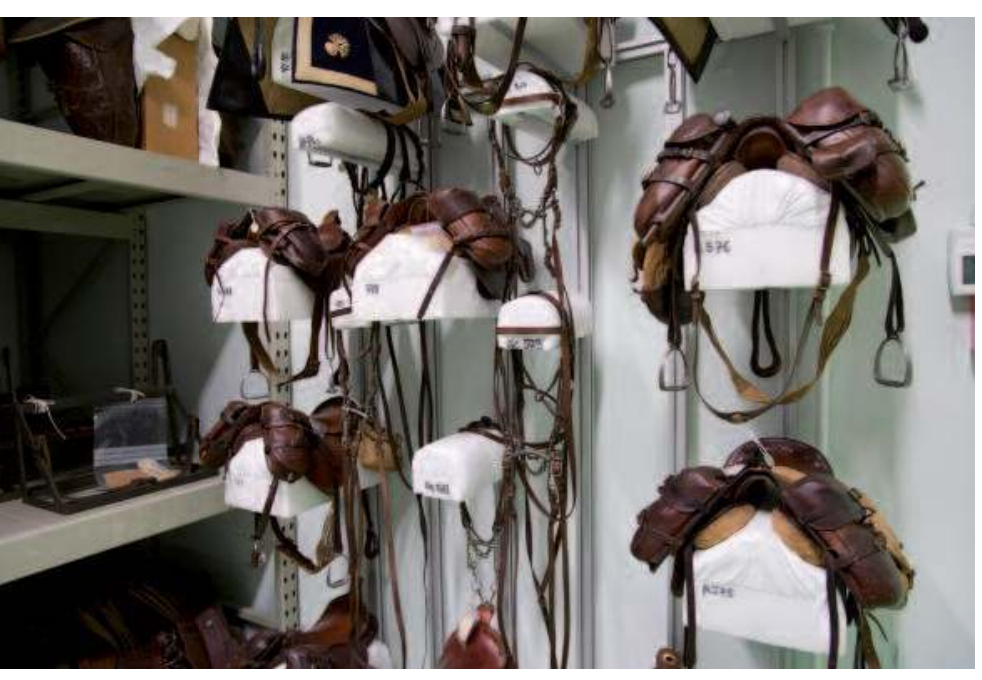

Les réserves: installation pour le stockage des selles ( ) Musée de l'Armée/Fanny Reynaud

sont effectuées scrupuleusement. Constat d'état des objets, identification, prise de mesures, prises de vues, dépoussiérage, marquage et conditionnement en sont les principales étapes. Tous les agents participent à différents niveaux. La personne chargée de l'entretien des réserves est tout aussi importante que le personnel de la conservation. Rien ne sert d'effectuer ces nombreuses opérations de conservation préventive si les objets sont ensuite stockés dans un environnement poussiéreux car peu ou pas entretenu. Les différents départements de la conservation sont présents quotidiennement dans les réserves pour assurer des travaux de recherche ou de récolement. Les objets sont localisés et les informations saisies sur le logiciel documentaire.

\section{Un règlement}

La réserve est un lieu hautement sécurisé, les procédures d'entrée sont strictes. Pour toute personne étrangère au musée, une demande d'autorisation d'accès doit être adressée à la direction et validée par le régisseur des collections. Les personnes sont accompagnées par un chargé de la surveillance pendant toute la durée de la visite. Les enregistrements des mouvements d'œuvres font l'objet d'une grande vigilance. Deux registres permettent leur traçabilité et la base documentaire renseignée. Il est interdit de boire ou manger à l'intérieur des salles de réserve.

\section{La lutte contre les biocontaminants}

Une veille sanitaire est observée sur les objets mensuellement. Les campagnes de piégeage des insectes sont organisées en permanence. Les pièges sont donc contrôlés régulièrement. Des sondages sont effectués sur différents objets stockés dans divers magasins de réserve afin de déceler au plus tôt d'éventuelles infestations. En cas de suspicion, l'objet est isolé dans l'aire de quarantaine afin d'être traité par anoxie statique, méthode maîtrisée par plusieurs personnels de la régie des collections. Tous les objets constitués de matériaux organiques qui entrent dans les réserves sont mis en quarantaine et traités selon la même méthode et cela de façon systématique. Enfin, les conditions climatiques sont contrôlées et enregistrées grâce à un système de capteurs thermohygrométriques électroniques qui équipent les trois bâtiments de réserves. Une analyse climatologique est effectuée tous les mois. De plus, un système d'alertes permet de prévenir en cas de dépassement des consignes demandées.

\section{L'avenir...}

La dernière tranche du plan de rénovation du musée s'achèvera en 2013 par la création aux Invalides d'un centre de documentation, un cabinet des estampes et une bibliothèque. Un chantier de ces collections vient de débuter et donnera lieu en 2012 à la naissance d'un nouveau bâtiment de réserve consacré à la conservation d'œuvres iconographiques. Sa capacité de stockage sera de $300 \mathrm{~m}^{2}$. Il sera équipé d'une centrale permettant le renouvellement d'air et d'une pompe à chaleur. Des espaces de travail permettront la consultation des documents et une salle sera dédiée à la restauration et équipée d'un matériel adapté mis à disposition des restaurateurs prestataires. Ce bâtiment est actuellement en cours d'étude par un cabinet d'architectes spécialisés en conservation préventive. À l'instar de ses trois aînés, ce bâtiment résultera d'un travail d'équipe, de l'association des diverses compétences dans des domaines variés et sa fonctionnalité dépendra de l'écoute et de l'intérêt portés aux remarques de ses futurs utilisateurs. 